Hydroécol. Appl. (1996) Tome 8 Vol. 1-2, pp. 85-98

\title{
Surveillance écologique et halieutique des sites de centrales nucléaires littorales: évaluation et perspectives
}

\section{Ecological and fishery monitoring of coastal nuclear power plants: assessment and prospects}

\author{
J.-Y. Quintin (1), F. Bordet(2)
}

(1) IFREMER Centre de Brest B.P. 70 - 29280 Plouzané Cedex. France.

(2) EDF Direction de l'équipement 30 av. de Wagram - 75382 Paris Cedex 08, France.

Résumé. - Électricité de France (EDF) a confié à l'Institut Français de recherche pour l'exploitation de la mer (IFREMER) la surveillance du milieu marin et de ses ressources vivantes autour des sites d'implantation de centrales nucléaires, installées le long du littoral français. Entreprises en 1974, les études écologiques se sont poursuivies sur les sites de Gravelines, Le Blayais, Paluel, Flamanville, et Penly, avec pour objectif d'appréhender l'impact de leur système de refroidissement en circuit ouvert, ainsi que de détecter les dérives éventuelles du milieu récepteur.

Pour répondre à cette attente, IFREMER a mis en place le programme Impact des Grands Aménagements (IGA). II a évolué en fonction des résultats acquis et permet sur la base d'un programme global, commun à tous les sites, d'intégrer les particularités de chacun d'entre eux. Sont abordés la nature et la problématique de l'impact, ainsi qu'une méthode d'approche et quelques résultats.

Au-delà de la réponse à la question de l'existence ou non d'un impact, les connaissances acquises sur l'évolution naturelle du milieu ont eu des retombées scientifiques. Elles permettent, en lien avec d'autres études de ce type, d'envisager une amélioration de la connaissance de notre environnement côtier, par l'observation, la compréhension et la gestion de ce milieu qui est le siege de conflits d'utilisation. En retour, une meilleure connaissance du milieu naturel devrait permettre de montrer si les effets observés au cours du temps ne sont pas le plus souvent le reflet de phénomènes naturels et, quand les effets de la centrale viennent se surajouter, de mieux gérer les situations limites pour l'écosystème.

Mots clés. - Études d'impact, Environnement marin, Gestion côtière. 


\begin{abstract}
Electricité de France (EDF) has asked Institut Français de recherche pour l'exploitation de la mer (IFREMER) to monitor the coastal marine environment and its living resources around nuclear power plants along the French coast. Started in 1974, these investigations were undertaken in the areas of Gravelines, Le Blayais, Paluel, Flamanville, and Penly; the objective is to detect any impact of the open-circuit water cooling system, as well as any differences in the marine environment itself.

To examine this question, IFREMER has set up a program called Impact des Grands Aménagements (IGA). It has evolved according to the results obtained and now integrates the specificity of each site in a global program common to all. The nature and the problems of impact are discussed, as well as the method of approach and some of the results.

Above and beyond the question of environmental impact, the information obtained on natural evolution of the coastal marine environment has had repercussions in scientific circles. Relating these results to similar studies, we can expect to improve our understanding through observation and management of this environment which gives rise to considerable conflicts in use. Furthermore, a better understanding of the natural coastal marine environment should enable us to determine whether the phenomena observed might not most frequently be the reflection of natural phenomena and when there is the added effect of power stations, how to manage situations critical for the ecosystem.
\end{abstract}

Key words. - Impact studies, Marine environment, Coastal management.

\section{INTRODUCTION}

Entrepris en 1974 à la suite des chocs pétroliers du début de décennie, un vaste programme de recherche de sites d'implantation de centrales électronucléaires de grande puissance sur le littoral français a provoqué un ensemble d'études écologiques du milieu marin et de ses ressources vivantes autour des sites potentiels d'implantation de Centres Nucléaires de Production d'Électricité (CNPE). Initiées par Electricité de France (EDF), ces études ont associé les grands organismes publics de recherche dont principalement le Centre National pour l'Exploitation des Océans (CNEXO) et l'Institut Scientifique et Technique des Pêches Maritimes (ISTPM), fusionnés depuis 1984 en Institut Français de Recherche pour l'Exploitation de la Mer (IFREMER), les Universités régionales, des laboratoires appartenant à diverses instances dont notamment l'Institut Pasteur de Lille, ainsi que les propres équipes d'EDF. De nombreux sites ont été prospectés dans le cadre d'études d'avant-projet: Gravelines et Paluel en 1974, Audinghen, Dannes, Penly, Englesqueville, Flamanville, Ploumoguer, Plogoff, St Vio, Corsept, Le Blayais en 1975, Vattetot, Beg-an-Fry, Erdeven, Brétignolles, Port-la-Nouvelle en 1976 (fig. 1). Ces études, effectuées avant la construction des centrales, ont contribué au choix de leur site d'implantation. Elles se sont poursuivies sous forme de projet, sur les sites retenus pour la construction des centrales de Gravelines, Le Blayais, Paluel, Flamanville, et Penly, pour affiner les connaissances écologiques et halieutiques. Elles conti- 


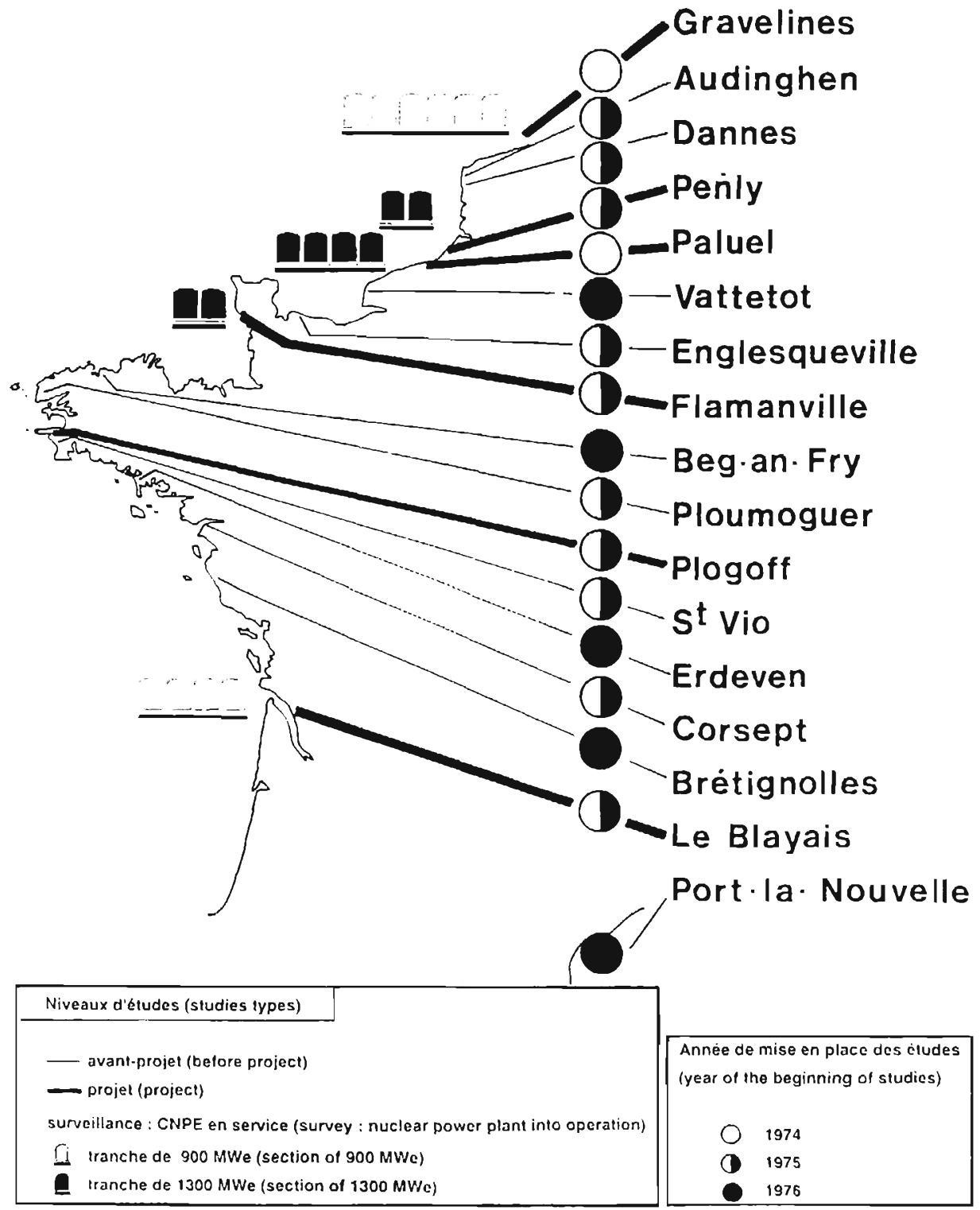

Fig. 1. - Etudes environnementales sur les sites littoraux de CNPE : niveaux d'études et annèe de leur mise en place.

Fig. 1. - Environmental studies of nuclear power plant: study levels and year of beginning. 
nuent maintenant dans un contexte de surveillance au cours de la phase d'exploitation.

\section{LES ÉTUDES DE SURVEILLANCE}

\subsection{Contenu des études}

Le principe d'étude de surveillance après la mise en service des centrales ayant été acquis dès le lancement du programme d'équipement, il restait à en définir le contenu.

L'axiome de base est qu' " on ne trouve généralement que ce que l'on cherche", d'où l'importance de définir, dès le départ, l'objectif recherché qui guide le choix de la stratégie de prélèvement et celui des paramètres indicateurs pertinents. Les connaissances disponibles à l'époque étaient éparses. II n'y avait pas de retour d'expérience, à l'exception de quelques études de sources étrangères ou de quelques résultats d'études sur les échauffements de l'eau et la chloration. Disponibles dans la bibliographie, ils ont été complétés par des travaux essentiellement de laboratoire sur le thème fédérateur de la thermoécologie (EDF, édit. 1977. 1981).

Les études de projet, par l'observation des cycles saisonniers des princıpaux parametres du milieu marin et des successions des especes biologiques ont permis de recueillir un maximum d'informations environnementales. Les bases d'un ètat de référence écologique et halieutique des sites. choisis par EDF. ont été éta- blies. Des paramètres indicateurs de qualité du milieu, des paramètres quantitatifs globaux (chlorophylle, biomasse zooplanctonique, densité...), des parametres descriptifs (principales espèces, indice de diversité...), des espèces-clés, caractéristiques d'un site et considérées comme sensibles à une modification éventuelle de leur milieu, ont été sélectionnés. La variabilité de leur abondance et de leur répartition dans le milieu a été estimée.

\section{2 Élaboration des programmes}

Lors de l'élaboration des programmes des études de surveillance, stratégies spatiale et temporelle ont été utilisées conjointement. L'une privilégie l'impact immédiat en considérant des comparaisons de type prise-rejet, elle intéresse une surface limitée aux abords immédiats des ouvrages; l'autre cherche à détecter des évolutions à moyen ou long terme du milieu marin par rapport à une situation de référence et recouvre une surface plus vaste à l'échelle iocale. A titre d'exemple pour le pélagos, une comparaison prise-rejet correspond á un impact immédiat de transit qui peut s'exprimer en terme de mortalité; une comparaison avant-après mise en service correspond à un impact différé qui peut se traduire par exemple par une évolution de l'équilibre entre espèces au sein d'une population.

Face à notre manque d'expérience, car les centrales nucléaires représentaient alors la première réalisation de surveillance d'un grand aménagement côtier, deux grandes idées ont guidé 
notre réflexion. Elles relèvent de façon plus ou moins explicite d'un principe de prudence, voire de précaution:

- Eviter que le choix trop restrictif de l'une des options, décidé à partir de considération a priori, élimine la possibilité de pouvoir détecter un évènement non prévu.

- Constituer des séries à long terme pour tenir compte des rythmes pluri annuels du milieu ce qui impose un choix initial pertinent des paramètres indicateurs.

\subsection{Choix de stratégie d'échantillonnage}

La solution retenue a donc été le résultat d'un compromis entre les deux options qui s'ouvraient à nous. Par exemple:

- Dans le cas du pélagos, celà s'est traduit par la définition d'un réseau de stations comprenant les stations prise, rejet, un point de contrôle dans la tache thermique, et un point hors impact, dit de référence. Le point de contrôle permet d'apprécier la tendance à un retour vers la normale par comparaison avec le point hors impact. Le point hors impact permet de détecter les fluctuations ou évolution du milieu "naturel».

- Dans le cas du benthos, la problématique étant voisine, la stratégie d'échantillonnage a été également le résultat d'un compromis entre le suivi des grandes zones biosédimentaires définies lors des études de projet et de l'étude quantitative détaillée de peuplements très localisés mais jugés sensibles.

Pour répondre à l'ensemble de cette attente, IFREMER a été amené à constituer des équipes, regroupant les compétences de spécialistes de toute thématique. Cela a permis de mettre progressivement en place un programme pluridisciplinaire sur l'Impact des Grands Aménagements (IGA). II a évolué en fonction des résultats acquis (Quintin \& Woehrling, 1993). II peut être considéré comme un test d'acuité pour la recherche à aborder l'enjeu $\mathrm{du} x \mathrm{xl}^{\mathrm{e}}$ siècle pour l'environnement, qu'est la protection du milieu marin, par son observation, sa compréhension, et sa gestion dans un contexte d'aménagement régional intégré.

\section{LA MÉTHODE D'ANALYSE STATISTIQUE}

\subsection{Approche par constat d'impact}

Sur la base des études de suivi temporel et de comparaison spatiale engagées dès le début des études, une première approche statistique a été réalisée pendant l'étude de l'état de référence du site de Gravelines. Elle a montré la possibilité de détecter statistiquement un écart entre un état de référence stable (avant mise en service) et un état impacté (après), en fonction du nombre d'années et de la valeur de la biomasse chlorophyllienne ${ }^{(1)}$ moyenne (Chardy \& Ménes-

(1) 15 ans avant et 15 ans après pour déceler un écart de $40 \%$ dans le cas d'une biomasse chlorophyllienne à $8 \mathrm{mg} / \mathrm{m}^{3}$, en période de bloom phytoplanctonique à Gravelines, calculé sur la base de 2 échantillons par point. 
guen, 1984). Cette approche se heurte en pratique au probleme du nombre très élevé (plusieurs dizaines par point de prélèvement) d'échantillons nécessaires pour discriminer de façon significative de faible écarts entre les états (avant-après) à analyser. L'étude statistique spatiale entre le milieu impacté et le milieu naturel apparaît plus réaliste. Cette approche par constat d'impact sur le milieu permet de calculer ou de constater un impact. Elle ne permet pas d'en saisir l'importance pour le milieu.

L'effort d'échantillonnage a été recentré sur le domaine spatial (milieu impacté-milieu naturel) à des périodes clés de l'année, tel qu'un bloom phytoplanctonique, un maximum zooplanctonique ou un maximun thermique annuel (fig. 2). En écologie pélagique pour le site de Flamanville par exemple, I'utilisation du test de Student (Erard-Le Denn et al., 1985), ou du test non paramétriques de Wilcoxon-MannWithney (Quintin et al., 1987) ont permis de faire apparaître, selon le paramètre étudié, les mois de l'année type où les variations du milieu sont les moins fortes. Le test de Kruskal-Wallis a permis de mettre en évidence des différences entre points; ce test est alors suivi d'un test de comparaison multiple pour identifier les points responsables de l'hétérogénéité spatiale lors des prélèvements (Quintin, 1989).

\subsection{Approche dynamique par analyse}

L'approche statique précédente se complète par une analyse écologique des effets observés, en particulier à partir des données recueillies en un point représentatif hors zone impactée. Ces données fournissent des informations sur l'état naturel des populations comme la richesse trophique, la productivité phytoplanctonique, l'abondance des stades larvaires réputés plus fragiles, l'âge des individus pour les œufs de poissons, etc.

C'est l'approche dynamique (Le Fèvre-Lehoerff \& Woehrling, 1991) qui permet de réajuster chaque année l'état de référence. Elle est fondée sur les connaissances scientifiques acquises, l'expérience des scientifiques impliqués, et doit être adaptée à chaque site. Elle permet de suivre l'évolution continuelle du milieu naturel dans le temps, tout en maintenant cette notion d'étude spatiale. Elle se situe dans le cadre d'une volonté d'optimisation du programme IGA, par la recherche de synergie avec d'autres programmes nationaux ou internationaux.

\section{VERS UNE VISION GLOBALE ET LOCALE}

Pour satisfaire ces objectifs, deux familles de paramètres sont échantillonnées.

La première famille permet de voir globalement l'état des lieux dans les domaines de l'écologie et de l'halieutique. Elle concerne des paramètres indicateurs tels que les caractéristiques des masses d'eau (température, salinité), les marqueurs de l'impact (température, haloformes), les sources 

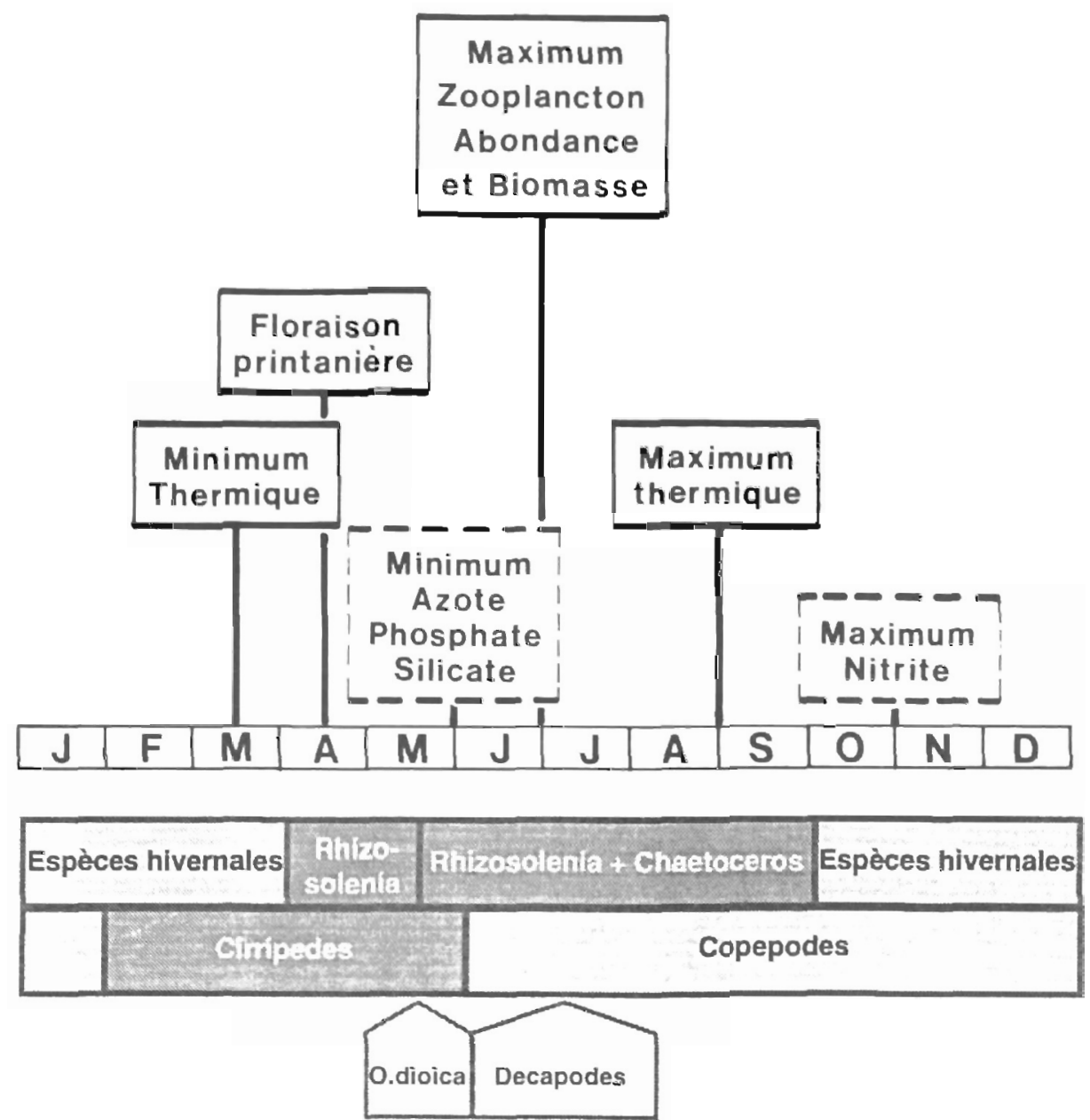

Fig. 2. - Schéma d'une année type en écologie pélagique à Flamanville (Manche orientale). Fig. 2. - Diagram of type year for pelagic ecology at Flamanville (East Channel).

nutritionnelles (sels nutritifs pour le phytoplancton, chlorophylle pour le zooplancton, zooplancton pour les poissons), les espèces et peuplements cibles (pélagiques ou benthiques), les espèces exploitées (benthos, halieutique).

La seconde famille comprend des paramètres indicateurs spécifiques de chaque site, sélectionnés pour leur sensibilité ou leur importance locale. Elle intéresse diverses thématiques tels que l'hydrologie (matière en suspension à Penly, bouchon vaseux au Blayais), la microbiologie (identification des Vibrions à Gravelines), la planctonologie (peuplements estuariens au Blayais), le benthos (Fucales 
à Paluel, Cirripèdes à Flamanville), la pêche expérimentale (homard et araignée à Flamanville), la biomorphosédimentologie (sonar latéral à Penly).

\section{RÉSULTATS PRINCIPAUX}

Quelques exemples permettent d'illustrer les résultats de ces programmes de surveillance et d'étude d'impact. Ils montrent souvent la difficulté que l'on rencontre lorsque l'on veut distinguer l'impact proprement dit (effet de transit, effet de chloration, effet d'échauffement), de ce qui relève de l'évolution naturelle du milieu marin, dans le temps et dans l'espace. Différents domaines sont concernés: l'écologie pélagique et benthique, l'halieutique, la morpho sédimentologie.

\subsection{Impacts immédiats}

Les impacts immédiats, essentiellement liés au transit dans les circuits, affectent particulièrement le phytoplancton, le zooplancton, et les juvéniles de poissons.

Les effets directs par courant de transit, parfois associés à un effet de chloration utilisée pour éliminer les salissures dans les circuits, ont été les premiers décelés. Dans le phytoplancton, une baisse de production primaire, correspondant à un déficit en biomasse chlorophyllienne, de 30 à $50 \%$ a été observé à Gravelines (Grossel, 1993).

Pour le zooplancton, une mortalité immédiate importante d'organismes mous, très fragiles, tels que l'espèce cible Oikopleura dioica à Gravelines a été observée. Le déficit en chlorophylle dans le panache échauffé étant également un facteur défavorable à cet herbivore, l'étude d'un éventuel effet différé du panache de rejet sur cette espèce se poursuit (Le FèvreLehoerff \& Woehrling, 1991).

Pour l'halieutique, une mortalité d'œuifs et larves de poissons a été constatée lors du transit, environ $30 \%$ d'œufs de Solea solea (Woehrling, 1983), ainsi que la capture par entrânement de poissons à Gravelines tels que le hareng Clupea harangus (Victor - Baptiste, 1984), et autres organismes planctoniques (Blanpied Wohrer, 1984).

\subsection{Impacts à long terme}

Les impacts à long terme peuvent être la conséquence d'un échauffement local de faible amplitude du milieu. Si l'observation d'effet de transit a prédominé, l'effet par échauffement du milieu récepteur est important à étudier, car il agit sur un paramètre fondamental dans les évènements climatiques à grande échelle. Le choix du type de circulation des eaux de refroidissement joue un rôle majeur dans la dispersion des eaux échauffées (fig. 3). Ils concernent par exemple la bactériologie, le zooplancton, le benthos.

L'optimum thermique des vibrions halophiles est élevé par rapport à la température naturelle de l'eau de mer: l'échauffement du rejet favorise leur prolifération, car les conditions 


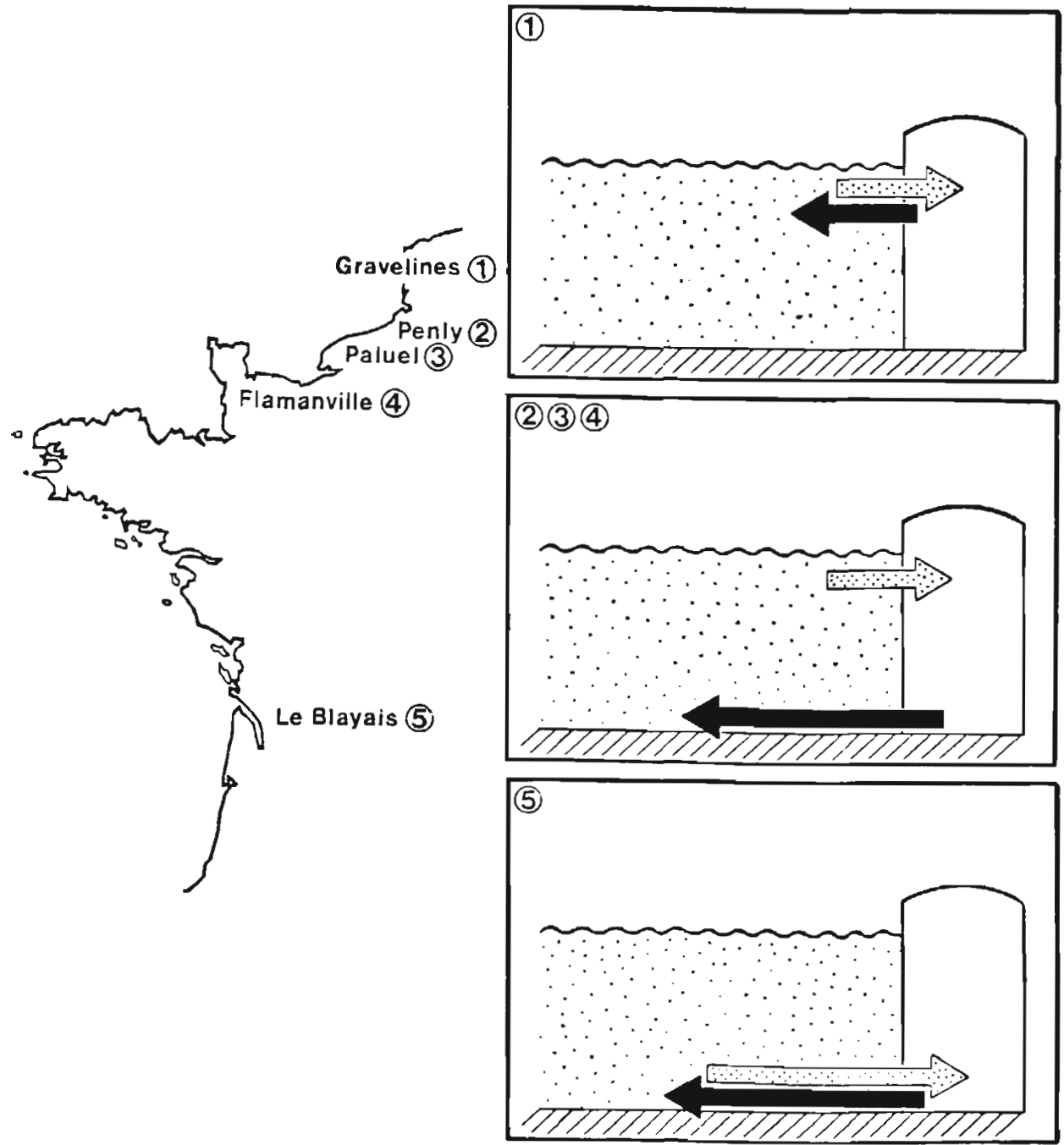

Fig. 3. - Différents types de circulation des eaux de refroidissement des sites de centre nucléaire de production d'électricité en bord de mer et en estuaire.

Fig. 3. - Various types of water cooling system of nuclear power plants on the coast and in an estuary.

optimales sont atteintes dans le rejet de la Centrale (Delesmont \& Delesmont, 1995). En effet les températures moyennes de l'eau, à Gravelines en été, sont proches de $20^{\circ} \mathrm{C}$ à l'entrée, et de $30^{\circ} \mathrm{C}$ au rejet.
Dans l'estuaire de la Gironde, une chute des effectifs du Copépode Eurytemora hirundoides a été observée en septembre-octobre 1991 au point impacté. Un lien a été établi avec son préférendum thermo-halin, schémati- 
sé par le diagramme de Bary (Castel, 1981). Les effets conjugués du rejet échauffé de la Centrale du Blayais et de conditions climatologiques naturelles exceptionnelles en 1991 (températures, pluies, débits, marées) en sont la cause (Castel, 1992).

Des perturbations ont été constatées sur les peuplements algaux à $\mathrm{Pa}$ luel, et sur le petit crustacé benthique Idotea granulosa qui s'y abrite (Richard \& Richard, 1995). Actuellement, un renforcement des études sur les fucus devrait permettre d'expliquer le phénomène, et pourrait éventuellement permettre de déterminer les raisons d'une modification de la fertilité dans le peuplement à Fucus serratus à Flamanville (Piriou, 1992; Drévès \& Piriou, 1994).

Les impacts à long terme peuvent aussi être la conséquences d'une modification courantologique avec ses répercussions sur la sédimentologie. Ils concernent par exemple le benthos et la morphologie sédimentaire.

Dans le benthos subtidal à Gravelines, le lessivage des fonds, provoqué par la circulation des eaux du rejet, a conduit à proximité de celui-ci à un appauvrissement du peuplement à Abra alba, où prédominent maintenant les espèces sabulicoles à régime alimentaire suspensivore (Dewarumez \& Menu, 1990a).

Dans le benthos intertidal à Gravelines, les effets conjugués de l'échauffement résiduel et de l'hydrodynamisme ont provoqué une modification topographique et sédimentologique de l'estran, accompa- gnée d'une redistribution de la répartition des espèces Pigospio elegans, annélide thermophile, et Spio martinensis, autre annélide (Dewarumez \& Menu, 1990b).

\subsection{Les retombées scientifiques}

Les retombées scientifiques du programme mis en place, par l'amélioration de la connaissance de l'environnement marin et estuarien et de la bande côtière, ont contribué à la réflexion d'ordre générale sur la notion d'impact à long terme, qui soulève en particulier le problème de la définition de l'état de référence servant de comparaison. En effet, on peut renconter :

- Soit un état moyen stable avec fluctuations autour de la moyenne. Les observations sont-elles alors contenues dans les fluctuations?

- Soit une tendance évolutive continue. Comment détecter et quantifier cette évolution naturelle?

Cela nous amène à souligner tout l'interêt que présente le suivi des séries chronologiques longues.

- La comparaison de plusieurs séries temporelles a permis de montrer des stuctures spatiales fortes entre la Mer du Nord et la Manche Ouest. (Le Fèvre-Lehoerff et al., 1993).

- L'évolution saisonnière de nombreux paramètres hydrologiques et biologiques d'un site peut permettre d'affiner la connaissance des cycles naturels, et de détecter éventuellement des évolutions climatiques (Fro- 
mentin \& Ibanez, 1994) et morphosédimentaires.

Le congrès du Programme National d'Océanographie Côtière (PNOC) d'Arcachon, en février 1995, a montré la difficulté de définir une période unique dans les fluctuations climatiques naturelles du milieu marin. II est nécessaire d'avoir une approche spatiotemporelle à long terme, sans négliger aucune échelle de temps.

Dans le cadre des études de surveillance du site de Gravelines, Woehrling et Le Fèvre-Lehoenff (1993) ont indiqué l'importance des années exceptionnelles pour les pontes de Sole et ont décelé l'existence d'un cycle de 7 ans sur ces espèces ichtyoplanctoniques Solea solea et Sprattus sprattus (Woehrling \& Le Fèvre-Lehoerff, soumis). Ce cycle existe aussi pour diverses espèces planctoniques Temora longicornis, Oikopleura dioica, Sagitta setosa. Un cycle de 11 ans apparaît pour Pseudocalanus minutus (Le Fèvre-Lehoerff et al., 1995). D'autres auteurs (Carpentier et al., 1997) ont également mis en évidence des cycles de 7 ans pour des espèces benthiques.

Les phénomènes observés peuvent être dus à des effets climatiques naturels exeptionnels dans lesquels la météorologie peut jouer un rôle. A Flamanville, la rigueur de l'hiver a un effet favorable sur le recrutement d'une espèce boréale, le Cirripède intertidal Balanus balanoides (Drévès, 1992), et les éclosions précoces de l'araignée de mer Maja squinado semblent liées à des températures printanières plus élévées que la moyenne (Martin, 1993).
Une augmentation progressive des températures minimales de l'eau de la Gironde s'observe sur le site du Blayais (Gironde, France) depuis le début des études en 1978. Mesurées au fond pour s'affranchir des fluctuations diurnes, elles sont corrélées à un accroissement progressif de la température de l'air (Castaing et al., 1995a). D'autres phénomènes lents sont observés, tel que l'évolution bathymétrique des fonds de l'estuaire (Castaing et al., 1995b). Tous ces phénomènes ont un effet sur la dispersion des eaux échauffées et le comportement de la flore et la faune locales, plus particulièrement lorsqu'une action anthropique vient se surajouter. Les effets peuvent être immédiats: c'est le cas de la chute de l'effectif d'Eurytemora en 1991.

\section{BILAN ET PERSPECTIVES}

Les études de surveillance du milieu marin au voisinage des centrales nucléaires se poursuivent depuis maintenant plus de quinze ans sur les sites les plus anciens. Ce recul permet, au delà des résultats scientifiques proprement dits, de tirer quelques enseignements de portée générale.

Les résultats scientifiques de ces études vérifient globalement les estimations prévisionnelles d'impact établies dans les différentes études d'impact produites lors des procédures administratives. Ils confirment que le fonctionnement des centrales 
n'entraîne actuellement pas d'altération quantitative notable du milieu marin pour les paramètres étudiés. De même ces résultats confortent les critères d'environnement retenus pour le choix des sites et l'intérêt pour des conceptions de circuits de refroidissement assurant une rapide dilution de l'effluent. A ce titre les différences entre Gravelines, où le rejet se fait à la côte en surface, et les autres sites bénéficiant d'un rejet au large en profondeur, apparaissent clairement en faveur de cette dernière disposition.

Du point de vue de la détection, voire de la quantification d'un impact modéré, ces études ont mis en évidence la difficulté à distinguer ce qui relève des fluctuations spatio temporelles du milieu, de ce qui est imputable aux rejets d'une centrale et cela justifie à postériori les principes qui avaient guidés EDF et IFREMER lors de l'élaboration de ces programmes. C'est-à-dire :

- l'importance de réaliser des séries chronologiques longues et de suivre, parallèlement aux stations impactées, une station de référence reflétant les variations naturelles du milieu;

- l'intérêt du bon choix initial des paramètres indicateurs, afin d'éviter les bouleversements notables du programme au cours de sa réalisation, et de bénéficier ainsi de séries chronologiques longues pour chaque paramètre retenu.

Une organisation et des moyens ont été mis en place pour répondre à la demande. La démarche du programme Impact des Grands Aménagements (IGA) répond à des objectifs opérationnels et de recherche. Ces objectifs sont la surveillance écologique et halieutique du milieu marin et estuarien, l'impact des installations et des rejets en construction et en fonctionnement, qui ne peuvent pas exister sans les compléter de la contribution à la connaissance objective de l'écosystème à court terme et à long terme.

Les enseignements tirés des études de surveillance permettent de quantifier le niveau des modifications, qui sont dues à "l'effet centrale" dans un milieu lui même fluctuant. Les résultats observés restent globalement en accord avec les prévisions effectués lors de la construction des centrales.

Pour aller plus loin, il faudrait pouvoir comprendre les phénomènes observés, et quand les phénomènes anthropogéniques se surajoutent aux phénomènes naturels, pouvoir mieux gérer les situations critiques pour l'écosystème. Par la recherche de synergie avec d'autres programmes, différentes périodicités ont été perçues dans les fluctuations naturelles du milieu marin. Elles sont à intégrer dans notre approche globale de la surveillance pour améliorer nos connaissances du milieu naturel lié aux sites, et des mécanismes qui le régulent. Elles nécessitent de disposer de séries chronologiques longues dont l'échelle de temps doit être adaptée aux différents paramètres étudiés. 


\section{RÉFÉRENCES BIBLIOGRAPHIQUES}

Blanpied-Wohrer C., 1984. Etude qualitative et quantitative des organismes entrainés dans la centrale de Gravelines (Nord). Influence des facteurs du milieu sur la capture de ces organismes. Thèse de $3^{\mathrm{e}}$ cycle, Université Pierre et Marie Curie, Paris VI, OcéanologieBiologie : $484 \mathrm{pp}$.

Castaing P., Jouanneau J.M., Oggian G. \& Severac J., 1995a. Climatologie et mesures in situ. Surveillance écologique du site du Blayais, année 1994, rapport scientifique. Rapport IFREMER pour EDF, DEL-95.08. : 25-94.

Castaing P., Jouannneau J.M., Oggian G. \& Severac J., 1995b. Evolution des fonds. Surveillance écologique du site du Blayais, année 1994, rapport scientifique. Rapport IFREMER pour EDF, DEL-95.08. : 97-101.

Chardy P. \& Menesguen A., 1984. Ecological monitoring and assessment in a coastal power plant study: alternative strategy. Thalassia Jugoslavia 20 (2) : 115-126.

Carpentier P., Dewarumez J.M. \& Leprêtre A., 1997. Long-term variability of the Abra alba community in the Southern Bight of the North Sea. Océanologica Acta, 20 (1) : 283-290.

Castel J., 1981. Aspect de l'étude écologique du plancton de l'estuaire de la Gironde. Océanis, 6 (6) : 535-577.

Castel J., 1992. Le zooplancton. Surveillance écologique du site du Blayais, année 1991, rapport scientifique. Rapport IFREMER pour EDF, DEL92.18. : 149-187 et 213-223.

Delesmont E. \& Delesmont R., 1995. Bactériologie et étude de la chloration. Surveillance écologique et halieutique du site de Gravelines. Rapport IFREMER pour EDF, DEL 95.07. : 17-37.

Dewarumez J.-M. \& Menu D., 1990a. Benthos subtidal. Etude de surveillance écologique et halieutique du site de
Gravelines (novembre 1988 - octobre 1989). Rapport IFREMER pour EDF, DRO-90.12-EL. : 97-120.

Dewarumez J.-M. \& Menu D., 1990b. Benthos intertidal. Etude de surveillance écologique et halieutique du site de Gravelines (novembre 1988 - octobre 1989). Rapport IFREMER pour EDF, DRO-90.12-EL. : 75-96.

Drévès L., 1992. Le domaine benthique. Surveillance écologique et halieutique du site de Flamanville, année 1991. Rapport IFREMER pour EDF, DEL/Brest-92.23. : 65-86.

Drévès L. \& Piriou J.-Y., 1994. Le domaine benthique. Surveillance écologique et halieutique du site de Flamanville, année 1993, rapport scientifique annuel. Rapport IFREMER pour EDF, DEL/Brest-94.09. : 51-74.

EDF, Direction de l'Equipement [édit.], 1977. Influence des rejets thermiques sur le milieu vivant en mer et en estuaire. Journées de la thermoécologie. Conférences et débats tenus les 15 et 16 novembre 1976 au Centre Océanologique de Bretagne (Brest). EDF Direction de l'équipement, Paris. $623 \mathrm{pp}$.

EDF, Direction de l'Equipement [édit.], 1981. Influence des rejets thermiques sur le milieu vivant en mer et en estuaire. $2^{\mathrm{e}}$ Journées de la thermoécologie. Conférences et débats tenus les 14 et 15 novembre 1979 à l'Institut Scientifique et technique des pêches maritimes à Nantes. EDF Direction de l'équipement, Paris. 882 pp.

Erard-Le Denn E., Gentien P. \& Quintin J.-Y., 1985. Paramètres synthétiques. Etude de surveillance écologique, site de Flamanville, année 1984. Rapport IFREMER pour EDF, DERO-86.12$E L: 2-14$.

Fromentin J.M. \& Ibanez F., 1994. Yearto-year changes in meterological feature of the French coast area during the last half-century. Examples of two biological responses. Oceanol. Acta, 17 (3) : 285-296. 
Grossel H., 1993. In: Le Fèvre-Lehoertf G., Grossel H., Woehrling D., Dewarumez J.-M. \& Delesmont R., 1993. La centrale nucleaire littorale de Gravelines, écologie, environnement et impact. J. Rech. Oceanogr., 18 (1 et 2) : 33-38.

Le Fèvre-Lehoerff G. \& Woehrling D., 1991. Aménagement industriel du littoral et surveillance de l'environnement: la centrale nucléaire de Gravelines (1975 à 1989). Actes du Colloque International sur l'environnement des Mers épicontinentales. Lille, 20-22 mars 1990. Oceanol. Acta, 11 : 299-311.

Le Fèvre-Lehoerff G., Erard-Le Denn E. \& Arzul G., 1993. Planktonic ecosystems in the Chanel. Trophic relations. Oceanol. Acta, 16 (5-6) : 661-670.

Le Fèvre-Lehoerff G., Ibanez F., Poniz P. \& Fromentin J.-M., 1995. Hydroclimatic relationships with planctonic time series from 1975 to 1992 in the North Sea off Gravelines, France. Mar. Ecol. Prog. Ser., Vol. 129 : 269-281.

Martin J., 1993. Période d'éclosion des larves d'araignée de mer Maja squinado Herbst en Manche et température de l'eau au printemps. - Cons. Int. Explor. Mer, Comité des mollusques et crustacés CM 1993/K: 50.

Piriou J.-Y., 1992. Phytobenthos intertidal. Surveillance écologique et halieutique, du site de Flamanville, année 1991. Rapport IFREMER pour EDF, DEL/Brest-92.23. : 53-62.

Quintin J.-Y., Gentien P. \& Erard-Le Denn E., 1987. Le domaine pélagique. Etude de surveillance écologique et halieutique, site de Flamanville, année 1985. Rapport IFREMER pour EDF, DRV-87.019-RH. DERO-87.28-EL : $13-75$.
Quintin, 1989. Zooplancton. In: le domaine pélagique. Surveillance écologique et halieutique, site de Flamanville, année 1987. Rapport IFREMER pour EDF, DRV-89.017-RH/Nantes, DEROEL / 89 12: 31-67.

Quintin J.-Y. \& Woehrling D., 1993. Centrales électronucléaires littorales et environnement: les études IGA. Equinoxe, 46 : 16-18.

Victor-Baptiste F., 1984. Etude qualitative et quantitative des organismes entraínés dans la centrale de Gravelines (Nord). Essai de quantification de l'impact global de la centrale: application aux harengs Clupea harengus $L$. These $3^{\ominus}$ cycle, Université Pierre et Marie Curie, Paris VI, OcéanologieBiologie : $442 \mathrm{pp}$.

Richard A. \& Richard I., 1995. Domaine benthique intertidal. Surveillance écologique et halieutique du site de Paluel, année 1994. Rapport IFREMER pour EDF.DEL/PB-95.02. : 79-106.

Woehrling D., 1983. Etude expérimentale de l'impact de la centrale de Gravelines (Nord) sur les œufs et larves de la sole (1982-1983). Rapport Institut Scientifique et Technique des Pêche Maritimes pour EDF.

Woehrling D. \& Le Fevre-Lehoerff G., 1993. Fluctuations pluriannuelles de la ponte de la sole Solea solea (L.) sur le littoral du sud de la Mer du Nord. J. Rech. Océanogr. 18 (3-4) : 74-79.

Woehrling D. \& Le Fevre-Lehoenf G. (soumis). Time-series in Ichtyoplancton: Sole and Sprat at Gravelines (French Coast of the North Sea) from 1975 to 1994. Comparison with other holoplanktonic and meroplanctonic groups. Actes du Colloque: les changements à long terme dans les écosystèmes marins. Arcachon 1-3 février 1995. Oceanol. Acta. (soumis). 